\title{
Refractive Index Sensor Based on Metal-Clad Planar Polymer Waveguide Operating at $850 \mathrm{~nm}$
}

\author{
Lanting JI, Wei WEI, Gang LI, Shuqing YANG, Yujie FU, Juan SU, and Chi WU* \\ Institute of Marine Science and Technology, Shandong University, Qingdao 266237, China \\ *Corresponding author: Chi WU E-mail: qi.wu@sdu.edu.cn
}

\begin{abstract}
A metal-clad planar polymer waveguide refractive index sensor based on epoxy (EPO) polymer materials by using light intensity interrogation at $850 \mathrm{~nm}$ is designed. The polymethyl methacrylate (PMMA) material is deployed as the low refractive index (RI) buffer layer in order to better couple the optical guided mode and the surface plasmon polaritons (SPP) mode for working in water environment. The effects of the gold film thickness, PMMA buffer layer thickness, waveguide layer thickness, waveguide width, and gold length on the sensor sensing characteristics have been comprehensively studied. Simulation results demonstrate that the normalized transmission increases quasi-linearly with the increment of RI of the analyte from 1.33 to 1.46 . The sensitivity is $491.5 \mathrm{~dB} / \mathrm{RIU}$, corresponding to a high RI resolution of $2.6 \times 10^{-9} \mathrm{RIU}$. The designed SPP-based optical waveguide sensor is low-cost, wide-range, and high-precision, and has a broad application prospect in biochemical sensing with merits of miniaturization, flexibility, and multiplexing.
\end{abstract}

Keywords: Metal-clad optical waveguide sensor; intensity interrogation; refractive index of liquid

Citation: Lanting JI, Wei WEI, Gang LI, Shuqing YANG, Yujie FU, Juan SU, et al., "Refractive Index Sensor Based on Metal-Clad Planar Polymer Waveguide Operating at 850 nm," Photonic Sensors, 2021, 11(4): 448-456.

\section{Introduction}

Refractive index (RI) is a critical parameter of liquid closely related to density, concentration, and temperature. The monitoring of RI of liquid is very important in fields of chemistry, medicine, physics, biology, and production practices. Surface plasmon polariton (SPP) is a kind of electromagnetic waves propagating along the metal-dielectric interface and has a sensitive response to the variation of RI of dielectric adjacent to the metal film $[1,2]$. The surface plasmon resonance (SPR) technology has now penetrated into the fields of food, biology, and environment, and demonstrated many advantages, such as high sensitivity, real-time detection, label-free, and selective recognition [3-7]. Due to the wavevector mismatch with optical waves, SPP waves are traditionally excited by using the Kretschmann or Otto prism based on attenuated total reflection, which has disadvantages of large volume, complex system, and high cost [8-10]. Then, the integrated optical waveguide technology is exploited to fabricate SPR sensor chips with advantages of low cost, small size, and easy integration [11-13]. The planar optical waveguide structure is used as the coupling channel, which avoids the complex prism system and is easy to interact with the external system. The waveguide-type SPR sensor can integrate the sensing area of the SPR with other semiconductor photoelectric devices to form a micro-on-chip detection system, which combines the manufacturing advantage of the silicon technology 
and the information transfer advantage of the optical waveguide. By optimizing the design, a better coupling between the optical guided mode and SPP mode can be achieved. In order to improve the performance of SPR sensors, many kinds of structures have been proposed, including polymer long-period waveguide grating with the liquid cladding [5], molecular imprinted based SPR sensor [7], and fiber-optic waveguide coupled SPR sensor [14]. Fan et al. [15] realized ultra-thin layer sensing by using a vertical SPP-dielectric waveguide coupler, and the detection sensitivity was as high as $0.67 \mathrm{~dB} / \mathrm{nm}$. Ma et al. [16] reported a biosensor based on the coupling between the long-range SPP waveguide and dielectric waveguide. The sensitivity was $4180 \mathrm{~nm} / \mathrm{RIU}$, and the detection limit was up to $3.7 \times 10^{-7}$ RIU. However, most of these works are done with wavelength interrogation and heavily rely on precise spectrometers and broadband optical sources, which are expensive, large-volume, and complex. This is difficult for low-cost, small-size, and portable monolithic integration. Compared with spectral interrogation, intensity interrogation becomes more and more portbale and practical. Especialy, with advances in laser diodes, photodetectors, and packaging technologies, SPR sensors based on intensity interrogation have demonstrated benefits of high resolution, high stability, easy integration, and multiplexing.

In this work, we present a waveguide-type SPR sensor based on epoxy (EPO) polymer materials by using light intensity interrogation at $850 \mathrm{~nm}$. The cost of light sources and detectors or cameras is low and aqueous solutions have a relatively low optical absorption at $850 \mathrm{~nm}$. The polymethyl methacrylate (PMMA) material is deployed as the low RI buffer layer to better couple the guided mode and the SPP mode in aqueous environment. The effects of the gold film thickness, PMMA buffer layer thickness, waveguide thickness, waveguide width, and gold length on the performance of the sensor have been comprehensively studied. Simulations demonstrate that the transmission increases quasi-linearly with the increment of liquid RI from 1.33 to 1.46 . The sensitivity of the sensor with an Au sensing length of $0.7 \mathrm{~mm}$ is $491.5 \mathrm{~dB} / \mathrm{RIU}$. The RI resolution is as high as $2.6 \times 10^{-9} \mathrm{RIU}$ in the analyte RI range from 1.33 to 1.46 , higher than those of the most SPR-based works that are on the $10^{-6}$ orders of magnitude [10-16].

\section{Structure and principle}

The metal-clad planar waveguide sensor is illustrated in Fig. 1. The waveguide consists of a rectangular core buried in a dielectric cladding on a silicon substrate. The width and height of the core are represented by $w$ and $h$, respectively. The gold (Au) film with a thickness of $t_{\mathrm{Au}}$ and a length of $L$ is deposited on the surface of the cladding. The $\mathrm{Au}$ film and the core are seperated by a PMMA buffer layer with a thickness of $t_{b}$. The PMMA is a high molecular compound polymerized by methyl methacrylate with merits of low cost, smooth surface, and high mechanical strength, and shows an excellent optical property of high transparency in a wide optical band. The RI of the PMMA is about 1.495, and that of waveguide core is about 1.577. The use of the PMMA buffer layer allows for a better coupling between the guided mode and SPP mode in aqueous environment (IR range 1.33 1.46). The commercial polymer EpoCore and EpoClad features of low cost, high stability, and easy integration are chosen as the waveguide materials [11]. The refractive indices of EpoCore, EpoClad, PMMA, Au, and the analyte are denoted by $n_{\mathrm{co}}, n_{\mathrm{cl}}, n_{b}, n_{\mathrm{Au}}$, and $n_{a}$, respectively. The analyte to be measured is liquid. When the guided mode propagates in the core, there exists the evanescent field in the cladding, and the SPPs mode is excited at the metal-analyte interface when satisfying the phase-matching condition. Due to the strong loss of SPPs, the coupling leads to a large waveguide mode loss. Even small variations in the RI of the analyte adjacent to the metal layer may result in large 
changes in the coupling strength and then the attenuation of the waveguide mode.

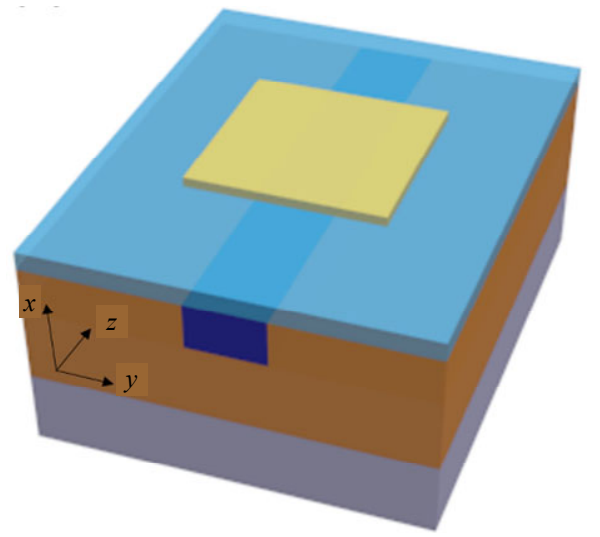

(a)

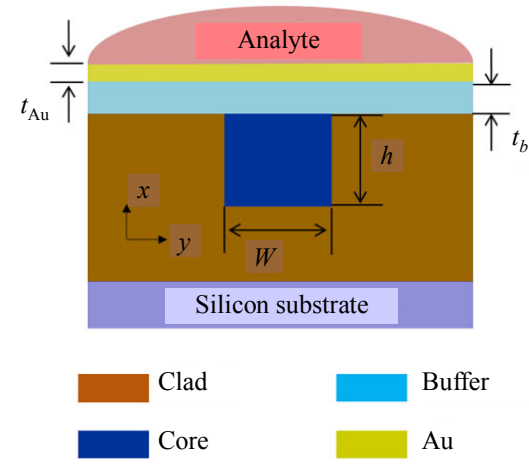

(b)

Fig. 1 Schematic diagram of (a) the 3-dimensional (3D) structure and (b) cross section of the designed RI sensor.

\section{Simulations}

For the metal-clad planar waveguide, there exists an optical guided mode in the core layer and a surface plasma mode at the interface between the metal and adjacent dielectric as well. The interaction between the guided mode and SPP mode can be analyzed by studying the optical properties of individual modes propagating in the sensing region $[11,15,17-19]$. When the guided mode is phase matched to the SPP mode, the electromagnetic fields of the mode are concentrated on the metal film of the structure, so it has a strong optical absorption. The RI sensor is theoretically designed by using the mode expansion and propagation method based on the finite element method [12]. The analysis of this configuration is based on the optical losses caused by the imaginary part of the complex propagation coefficient, which is related to the imaginary part of the effective indice of the guided mode. The modal absorption is derived from the complex propagation coefficient for the transverse magnetic (TM) modes of the structure, as a function of RI of the analyte. Firstly, the mode analysis is performed on the cross section of the optical metal-clad planar waveguide to calculate the roots of the eigenvalue equation. Then, according to the calculation results, the dispersion characteristics of the TM fundamental guided mode and SPP mode are extracted. The refractive indices are assumed as follows: $n_{\mathrm{co}}=1.577, n_{\mathrm{cl}}=1.565$, and $n_{b}=1.495$. The variation of complex permittivity of $\mathrm{Au}$ with the wavelength is taken from the published experimental data [20]. Compared with the strong variation of complex permittivity of the gold film with the wavelength, material dispersion effects on the other media are negligible here. Because only the TM polarization waveguide mode can be coupled to the SPP mode, in the following simulations, we consider only TM modes.

Firstly, we calculate the dispersion properties of the individual guided mode and SPP mode based on the finite element method. The real and imaginary parts of effective RI of the waveguide are denoted by $\operatorname{Re}\left(N_{\text {eff }}\right)$ and $\operatorname{Im}\left(N_{\text {eff }}\right)$, respectively. Figure 2 shows the variation of $\operatorname{Re}\left(N_{\text {eff }}\right)$ with the wavelength. The structural parameters used here are as follows: $w=h=3 \mu \mathrm{m}, t_{\mathrm{Au}}=50 \mathrm{~nm}, t_{b}=500 \mathrm{~nm}$, and $n_{a}=1$. It is clear that the real parts of effective RI of the guided mode and SPP mode cross each other at $855 \mathrm{~nm}$, so the propagation constants of the guided mode and SPP mode match well at the intersection point of $\lambda=855 \mathrm{~nm}$. In the implementation of the experiment, the fundamental TM mode could be excited in the input waveguide by the end-fire coupling method. To reduce the coupling loss, the tapered cylindrical optical fiber can be used. The fiber-waveguide energy coupling efficency mainly depends upon the overlap between the core mode 
and fiber mode. The power emerging from the sensing waveguide $P_{\text {out }}$ is exoressed as [21]

$$
P_{\text {out }}=P_{\text {in }} C^{2} \mathrm{e}^{-2 \alpha L_{\text {Au }}}
$$

where $P_{\text {in }}$ is the input power from the fiber, $C$ is the end-fire coupling factor, $\alpha=2 \pi \operatorname{Im}\left(N_{\text {eff }}\right) / \lambda$ is the waveguide attenuation coefficient, and $L_{\mathrm{Au}}$ is the length of the gold area. The insertion loss $(I L)$ of the sensor is simplied as [21]

$$
I L=-20 \lg (C)+L_{\mathrm{Au}} \cdot M P A
$$

where $M P A=40 \pi \lg (\mathrm{e}) \operatorname{Im}\left(N_{\text {eff }}\right) / \lambda$ is the mode power attenuation of the waveguide. In this work, the normalized transmission is defined as the absorption loss caused by the metal-clad waveguide, excluding the fiber-waveguide end coupling loss.

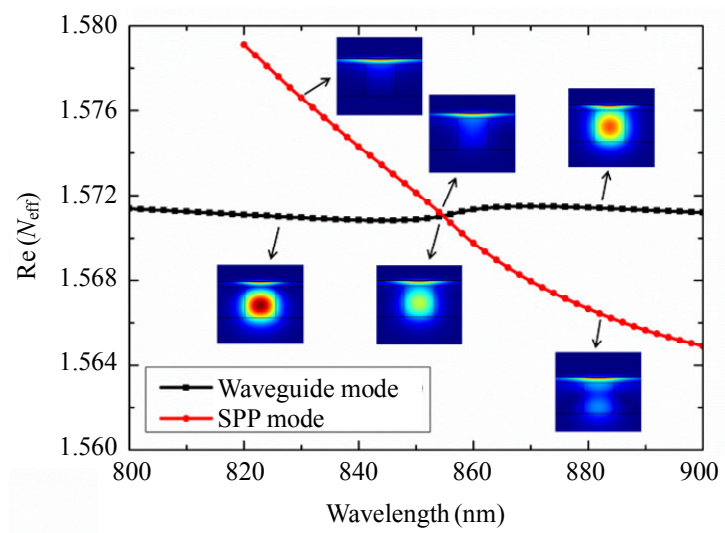

Fig. 2 Dispertion curves of the waveguide and SPP mode when $w=h=3 \mu \mathrm{m}, d_{\mathrm{Au}}=50 \mathrm{~nm}$, and $t_{b}=500 \mathrm{~nm}$.

The normalized transmission of the device as a function of the wavelength with different external analytes is shown in Fig. 3. There exists a resonance wavelength at $855 \mathrm{~nm}$ with an extinction ratio of $-72 \mathrm{~dB}$ when $n_{a}$ is 1.33 . When $n_{a}$ incerases from 1.33 to 1.36 , the maximum absorption wavelength shifts to a longer wavelength. For a fixed operation wavelength of $850 \mathrm{~nm}$, the transmission power increases $\Delta P$, which can be detected by an optical power meter. The SPR sensor described here is designed to operate around $\lambda=850 \mathrm{~nm}$, which corresponds to a central wavelength of the commercially available laser source. The cost of light sources and detectors or cameras at $850 \mathrm{~nm}$ is low that facilitates low-cost and portable sensing applications. And those aqueous solutions with analytes have a relatively low optical absorption at $850 \mathrm{~nm}$ [22, 23]. Varying RIs of analytes, the variation of output power is recorded in real time from the output waveguide. Compared with the spectral interrogation, light intensity interrogation shows advantages of fast response, low cost, and easy integration.

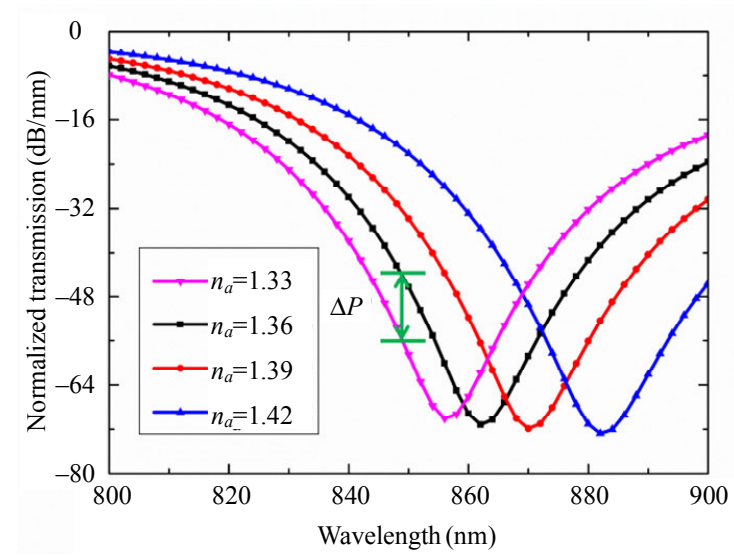

Fig. 3 Normalized transmission as a function of the wavelength when $w=h=3 \mu \mathrm{m}, d_{\mathrm{Au}}=50 \mathrm{~nm}$, and $t_{b}=500 \mathrm{~nm}$.

Figure 4 shows the variation of effective RI of the guided mode with a waveguide width when $h$ is $3.2 \mu \mathrm{m}$. Single mode operation can be achieved within a certain waveguide width from $1 \mu \mathrm{m}$ to $3.5 \mu \mathrm{m}$. Therefore, the width of the waveguide $w$ is set to be $3.2 \mu \mathrm{m}$.

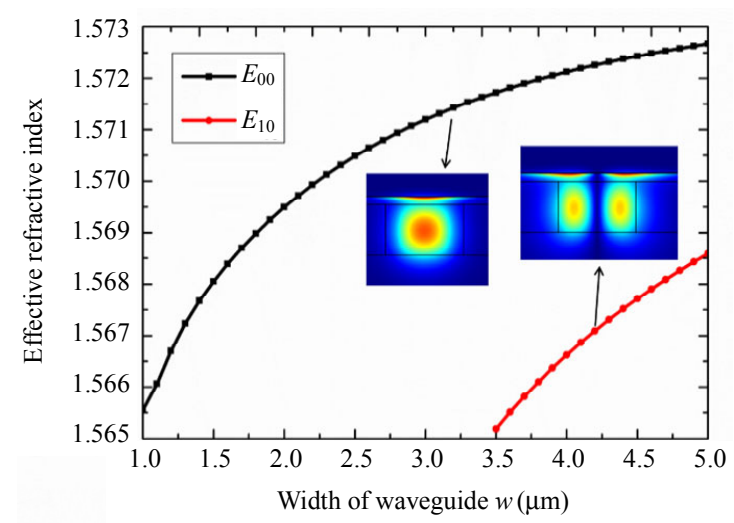

Fig. 4 Effective refractive index of the waveguide mode changes with the core width from $1.0 \mu \mathrm{m}$ to $5.0 \mu \mathrm{m}$.

To realize the best phase matching condition at the wavelength of $850 \mathrm{~nm}$, the thicknesses of the PMMA buffer layer and gold film are optimized. The normalized transmission of the device versus the thickness of the PMMA buffer layer with $h=$ 
$3.2 \mu \mathrm{m}, w=3.2 \mu \mathrm{m}, t_{\mathrm{Au}}=50 \mathrm{~nm}$, and $n_{a}=1.33$ is calculated, as shown in Fig. 5(a). The loss firstly increases to a maximum value of $-60 \mathrm{~dB}$ when $t_{b}$ is around $500 \mathrm{~nm}$ and then decreases, which implies that when $t_{b}$ is about $500 \mathrm{~nm}$, the coupling efficiency reaches the peak. Since the thickness of gold is directly related to the amount of free electron charge, it has a significant effect on the mode peak attenuation and SPR dip position. Parameters used here are as follows: $w=3.2 \mu \mathrm{m}, h=3.2 \mu \mathrm{m}, t_{b}=$ $500 \mathrm{~nm}$, and $n_{a}=1.33$. As shown in Fig. $5(\mathrm{~b})$, the

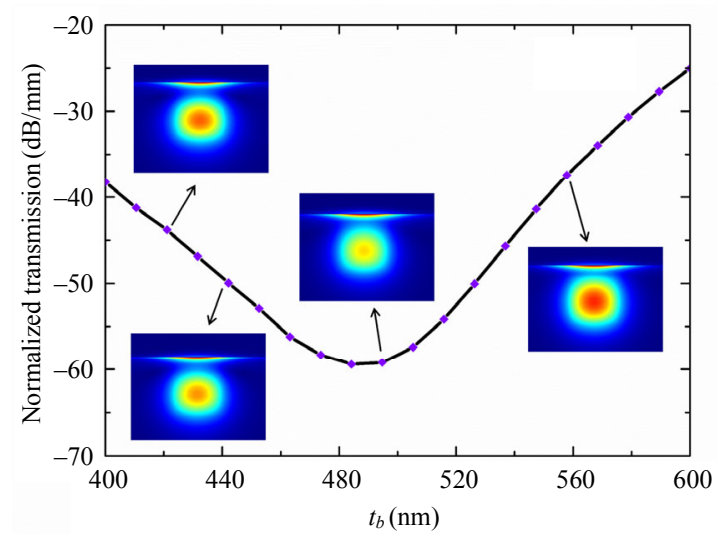

(a) loss firstly increases to a maximum value of $-68 \mathrm{~dB}$ when $t_{\mathrm{Au}}$ is $51.5 \mathrm{~nm}$ and then decreases. The insets show the mode field distributions of the waveguide at different $\mathrm{Au}$ film thicknesses. The mode distributions decay in the waveguide core and most locate in the $\mathrm{Au}$ layer when $t_{\mathrm{Au}}$ is $51.5 \mathrm{~nm}$, while most of the mode fields distribute in the waveguide core when $t_{b}$ is $42 \mathrm{~nm}$. Due to a large absorption by the metal, the mode attenuation in the waveguide with the $51.5 \mathrm{~nm}$ thick gold film is greater than those with other thick gold films.

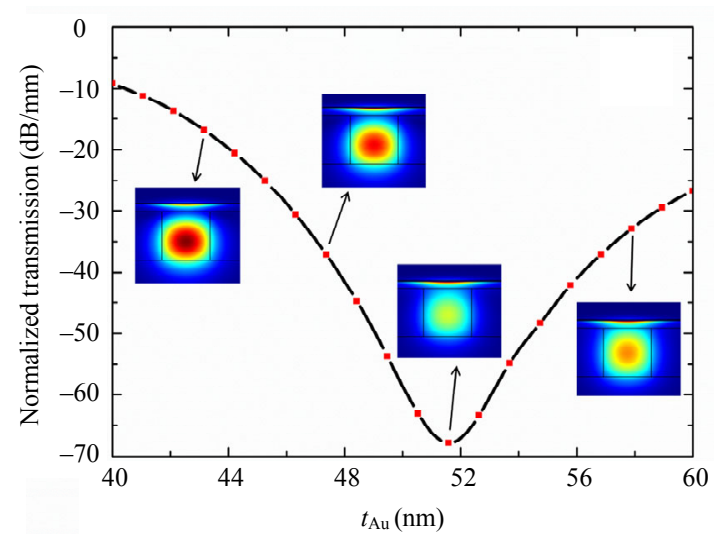

(b)

Fig. 5 Normalized transmission changes (a) with the thickness of the PMMA buffer layer and (b) with the thickness of the gold film.

\subsection{Effects of thickness of the gold film}

In the design of the structure, it is found that the maximal transmission loss happens when the gold film thickness is around $51.5 \mathrm{~nm}$. The influence of the Au thickness on the sensing characteristics is investigated. Figure 6 shows the dependence of the normalized transmission on RI of the analyte for different gold film thicknesses of $46 \mathrm{~nm}, 48 \mathrm{~nm}$, $50 \mathrm{~nm}$, and $51.5 \mathrm{~nm}$. The structural parameters used here are as follows: $w=h=3.2 \mu \mathrm{m}, t_{b}=500 \mathrm{~nm}, \lambda=$ $850 \mathrm{~nm}$, and $L_{\mathrm{Au}}=1 \mathrm{~mm}$. It is clear that the transmission increases quasi-linearly with the increment of RI of the analyte and the sensitivity of the sensor changes significantly with the variation of the Au thickness. As the metal becomes thicker from $46 \mathrm{~nm}$ to $51.5 \mathrm{~nm}$, the sensitivity increases about 2.5 -fold from $187.81 \mathrm{~dB} / \mathrm{RIU}$ to $466.49 \mathrm{~dB} / \mathrm{RIU}$. The variation of the thickness of the gold film significantly affects the dispersion property of the SPP mode and also affects the coupling between the guided mode and SPP mode.

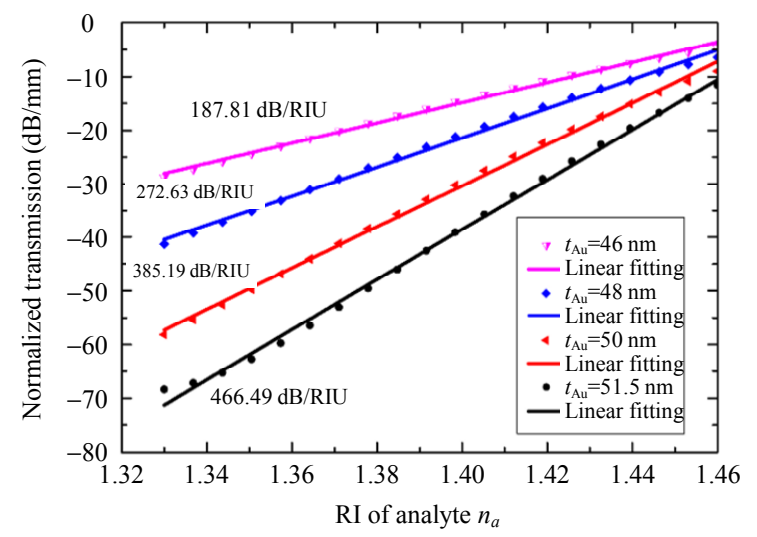

Fig. 6 Dependence of the normalized transmission on RI of the analyte for different gold film thicknesses.

\subsection{Effects of the thickness of the PMMA layer}

The influence of variation of the PMMA thickness on the sensor is investigated. From 
Fig. 5(b), the transmission loss reaches its maximum when $t_{b}$ is around $500 \mathrm{~nm}$. Similarly, we select $460 \mathrm{~nm}, 480 \mathrm{~nm}$, and $500 \mathrm{~nm}$ thick buffer layers for simulation. Figure 7 depicts the variation of normalized transmission with the increment of RI of the analyte under the condition that $w=h=3.2 \mu \mathrm{m}$, $t_{\mathrm{Au}}=50 \mathrm{~nm}, \lambda=850 \mathrm{~nm}$, and $L_{\mathrm{Au}}=1 \mathrm{~mm}$. As the thickness of the buffer layer increases from $420 \mathrm{~nm}$ to $500 \mathrm{~nm}$, the sensitivity increases from $252.9 \mathrm{~dB} / \mathrm{RIU}$ to $385.19 \mathrm{~dB} / \mathrm{RIU}$ in the RI range of $1.33-1.46$ to be measured. The variation of the thickness of the PMMA layer changes the effective index of the two modes, which in turn affects the phase matching condition.

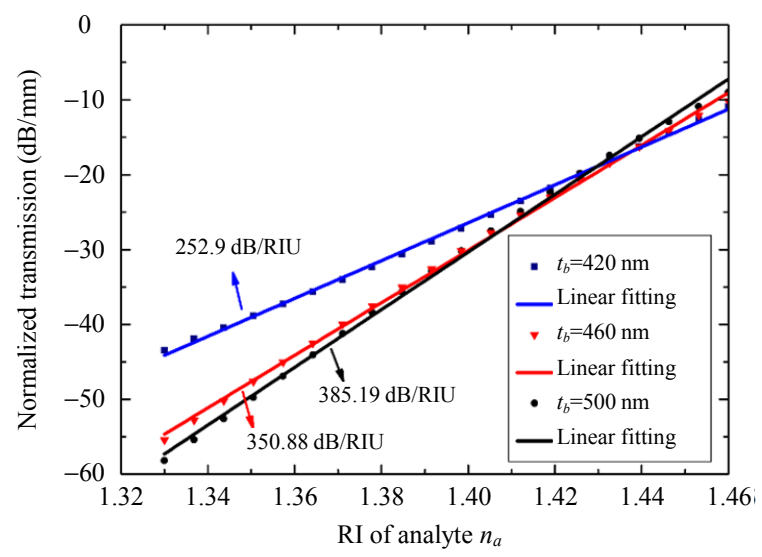

Fig. 7 Dependence of the normalized transmission on RI of the analyte for different thicknesses of the buffer layer.

\subsection{Effects of the width and thickness of the waveguide}

The normalized transmission changes with the external refractive index for different waveguide widths and different waveguide thicknesses are studied, as shown in Figs. 8 (a) and 8(b). Figure 8(c) shows the sensitivity of the sensor varies with the waveguide width and waveguide thickness. The structural parameters are as follows: $t_{b}=500 \mathrm{~nm}, \lambda=$ $850 \mathrm{~nm}, t_{\mathrm{Au}}=50 \mathrm{~nm}$, and $L_{\mathrm{Au}}=1 \mathrm{~mm}$. The sensitivity increases slightly from $354 \mathrm{~dB} / \mathrm{RIU}$ to $402 \mathrm{~dB} / \mathrm{RIU}$ when the waveguide width increases from $2.8 \mu \mathrm{m}$ to $3.4 \mu \mathrm{m}$, because the interaction width between the guided mode and SPP mode, as well as the interaction width between the electric field and external medium, increases. When $h$ increases from $2.6 \mu \mathrm{m}$ to $3.4 \mu \mathrm{m}$, the sensitivity decreases from

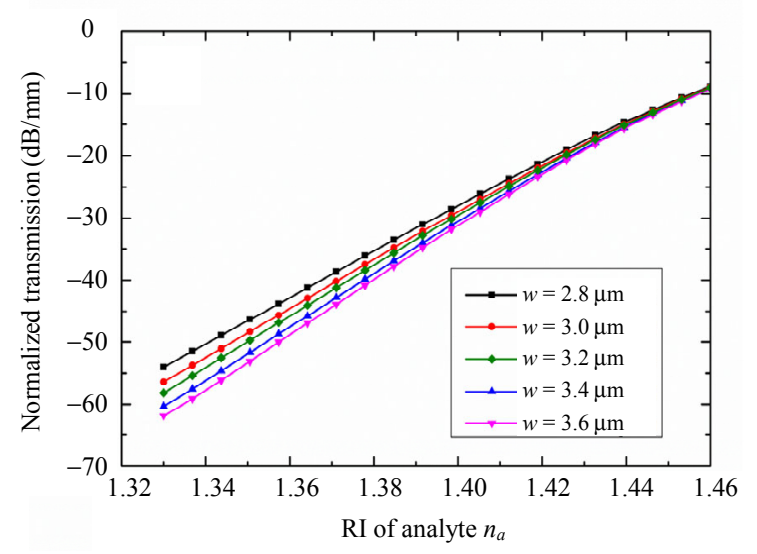

(a)

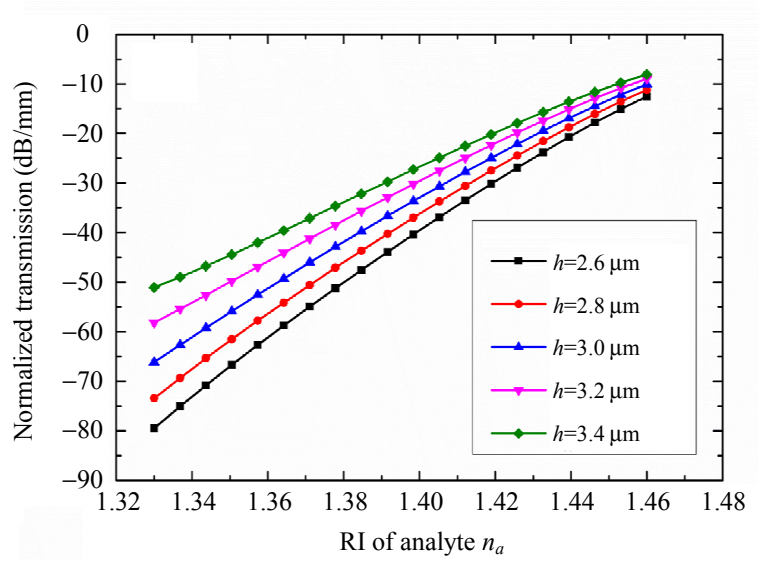

(b)

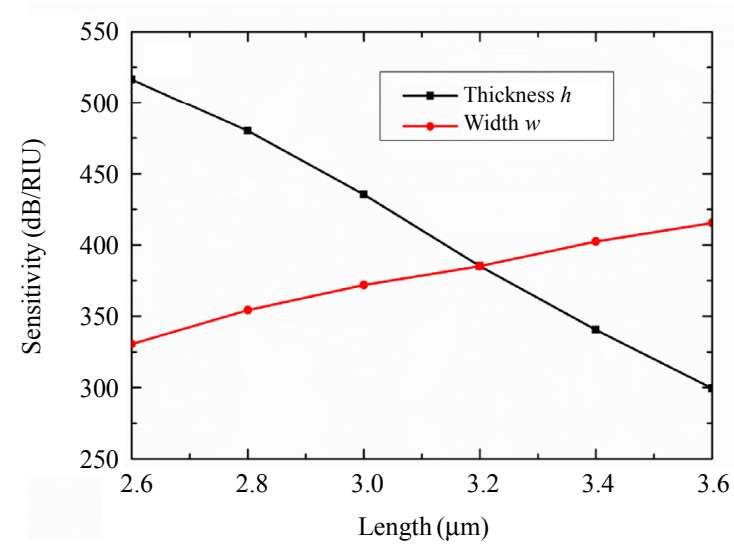

(c)

Fig. 8 Dependence of the normalized transmission on RI of the analyte for different (a) waveguide widths, $h=3.2 \mu \mathrm{m}$, (b) waveguide thicknesses, $w=3.2 \mu \mathrm{m}$, and (c) the sensitivity of the sensor varies with the waveguide width and waveguide thickness. 
$516.19 \mathrm{~dB} / \mathrm{RIU}$ to $340.44 \mathrm{~dB} / \mathrm{RIU}$. The thinning of the waveguide results in an increase in the electric field density of the guided layer that strengthens the interaction between the electric field and external medium. Compared with the width of the waveguide, the variation of the waveguide thicknesses has more influence on the sensitivity of the sensor, since the SPP waves are TM polarized.

\subsection{Effects of length of the Au sensing area}

Figure 9 shows the normalized transmission changes with RI of the analyte for different $\mathrm{Au}$ lengths of $0.2 \mathrm{~mm}, 0.6 \mathrm{~mm}$, and $1 \mathrm{~mm}$. The structural parameters are as follows: $w=h=3.2 \mu \mathrm{m}, t_{\mathrm{Au}}=$ $50 \mathrm{~nm}, \lambda=850 \mathrm{~nm}$, and $t_{b}=500 \mathrm{~nm}$. As can be seen, the sensitivity increases 5 -fold from $77.04 \mathrm{~dB} / \mathrm{RIU}$ to $385.19 \mathrm{~dB} / \mathrm{RIU}$ as the sensing length increases from $0.2 \mathrm{~mm}$ to $1.0 \mathrm{~mm}$, while the transmission loss increases as well. Therefore, there exists a compromise between the sensitivity and the loss when optimizing the length of the Au sensing area.

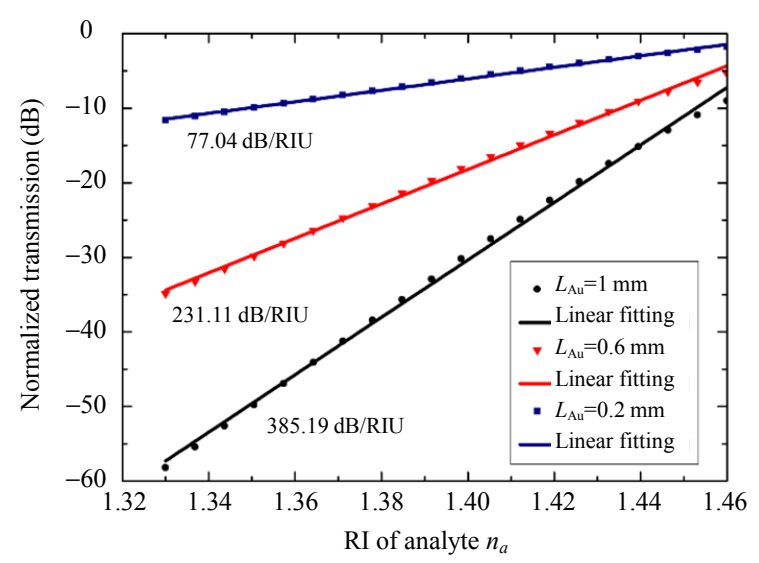

Fig. 9 Dependence of normalized transmission on RI of analytes for different sensing lengths of $1 \mathrm{~mm}, 0.6 \mathrm{~mm}$, and $0.2 \mathrm{~mm}$.

\section{Results and discussion}

According to the above simulations, the parameters are selected as follows: $w=3.2 \mu \mathrm{m}, h=$ $2.8 \mu \mathrm{m}, d_{\mathrm{Au}}=52 \mathrm{~nm}, t_{b}=500 \mathrm{~nm}$, and $L_{\mathrm{Au}}=0.7 \mathrm{~mm}$. The imaginary part of RI of water at $850 \mathrm{~nm}$ is around $2.8 \times 10^{-7}$ [22]. Considering the absorption of light by water, the dependence of normalized transmission on RI of the analyte is shown in Fig. 10. When RI of the analyte increases from 1.33 to 1.46 , the transmission increases quasi-linearly from $-75 \mathrm{~dB}$ to $-10 \mathrm{~dB}$, corresponding to a sensitivity of $491.5 \mathrm{~dB} / \mathrm{RIU}$. If the power range of the photodetector is from $500 \mathrm{pW}$ to $5 \mathrm{~mW}$, and the resolution is $100 \mathrm{pW}$ (PM130D, Thorlab, America), the detection limit of the RI sensor is as high as $2.6 \times 10^{-9} \mathrm{RIU}$, improved by at least two orders of magnitude compared with those of the SPR sensors based on the spectral interrogation [8-12].

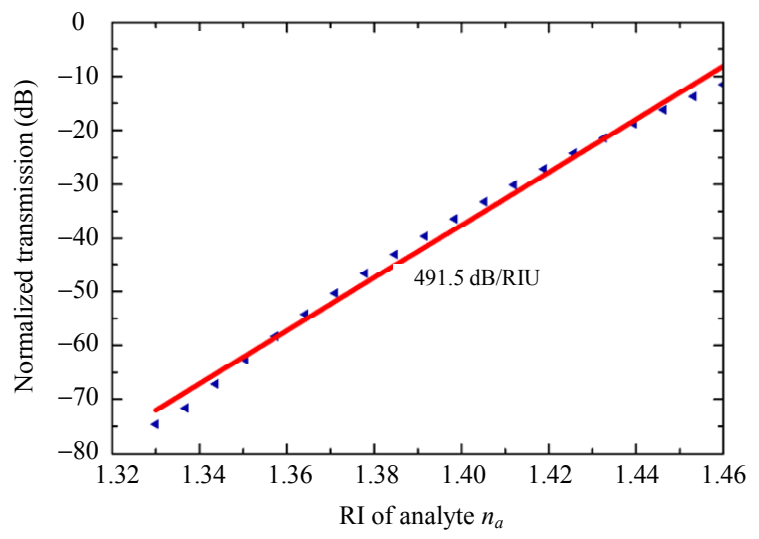

Fig. 10 Normalized transmission changes with RI of the analyte.

\section{Conclusions}

A metal-clad planar waveguide SPR sensor based on EPO polymer materials by using light intensity interrogation at $850 \mathrm{~nm}$ is demonstrated. The effects of the gold film thickness, PMMA buffer layer thickness, waveguide layer thickness, waveguide width, and sensing length on sensing performance of the RI sensor have been comprehensively studied. The normalized transmission increases quasi-linearly with the increment of RI of the analyte. The sensitivity changes significantly with the variation of the $\mathrm{Au}$ thickness, since the thickness of the gold film directly affects the amount of free electron charge. The sensing sensitivity increases with the decrement of the waveguide thickness. The sensitivity of the sensor is $491.5 \mathrm{~dB} / \mathrm{RIU}$ in RI range of $1.33-1.46$ 
for the $\mathrm{Au}$ sensing length of $0.7 \mathrm{~mm}$ in the 52-nm-thick Au layer, 500-nm-thick PMMA layer, and $3.2 \times 2.8 \mu \mathrm{m}^{2}$ waveguide, corresponding to a high RI resolution of $2.6 \times 10^{-9}$. A higher sensitivity can be achieved by optimizing the waveguide geometric parameters and materials. The designed hybrid waveguide coupled SPPs-based liquid RI sensor is low-cost, compact, wide-range, and high-precision, and shows a broad application prospect in biochemical and environment sensing fields.

\section{Acknowledgment}

The authors gratefully acknowledge the financial support from the Shandong Provincial Key Research and Development Program (Grant Nos. 2018YFJH0702 and 2019JZZY020711), Shandong Postdoctoral Innovation Project, and Qingdao Postdoctoral Applied Research Project.

Open Access This article is distributed under the terms of the Creative Commons Attribution 4.0 International License (http://creativecommons.org/licenses/by/4.0/), which permits unrestricted use, distribution, and reproduction in any medium, provided you give appropriate credit to the original author(s) and the source, provide a link to the Creative Commons license, and indicate if changes were made.

\section{References}

[1] W. L. Barnes, A. Dereux, and T. W. Ebbesen, "Surface plasmon subwavelength optics," Nature, 2003, 424(6950): 824-830.

[2] V. V. Temnov, G. Armelles, U. Woggon, D. Guzatov, A. Cebollada, A. Garcia-Martin, et al., "Active magneto-plasmonics in hybrid metal-ferromagnet structures," Nature Photonics, 2010, 4(2): 107-111.

[3] K. Brahmachari, S. Ghosh, and M. Ray, "Surface plasmon resonance based sensing of different chemical and biological samples using admittance loci method," Photonic Sensors, 2013, 3(2): 159-167.

[4] M. Xue, Q. Jiang, C. Zhang, and J. Lin, "A kind of biomolecular probe sensor based on TFBG surface plasma resonance," Photonic Sensors, 2015, 5(2): 102-108.

[5] L. Wang, K. Ren, B. Sun, and K. Chen, "Highly sensitive refractive index sensor based on polymer long-period waveguide grating with liquid cladding," Photonic Sensors, 2019, 9(1): 19-24.

[6] Q. Jiang, M. Xue, P. Liang, C. Zhang, J. Lin, and J. Ouyang, "Principle and experiment of protein detection based on optical fiber sensing," Photonic Sensors, 2017, 7(4): 317-324.

[7] D. Çimen and A. Denizli, "Development of rapid, sensitive and effective plasmonic nanosensor for the detection of vitamins in infact formula and milk samples," Photonic Sensors, 2020, 10(4): 316-332.

[8] R. K. Verma, A. K. Sharma, and B. D. Gupta, "Surface plasmon resonance based tapered fiber optic sensor with different taper profiles," Optics Communications, 2008, 281(6): 1486-1491.

[9] Y. Yuan, N. Yuan, D. Gong, and M. H. Yang, "A high-sensitivity and broad-range SPR glucose sensor based on improved glucose sensitive membranes," Photonic Sensors, 2019, 9(4): 309-316.

[10] D. Michel, F. Xiao, and K. Alameh, "A compact, flexible fiber-optic Surface Plasmon Resonance sensor with changeable sensor chips," Sensors and Actuators B: Chemical, 2017, 246: 258-261.

[11] S. K. Mishra, B. Zou, and K. S. Chiang, "Surface-plasmon-resonance refractive-index sensor with Cu-coated polymer waveguide," IEEE Photonics Technology Letters, 2016, 28(17): 1835-1838.

[12] J. Dostálek, J. Čtyroký, J. Homola, E. Brynda, M. Skalský, and P. Nekvindová, "Surface plasmon resonance biosensor based on integrated optical waveguide," Sensors and Actuators B: Chemical, 2001, 76(1-3): 8-12.

[13] L. Ji, X. Sun, G. He, L. Yu, X. Wang, Y. Yi, et al., "Surface plasmon resonance refractive index sensor based on ultraviolet bleached polymer waveguide," Sensors and Actuators B: Chemical, 2017, 244: 373-379.

[14] J. H. Ahn, T. Y. Seong, W. M. Kim, T. S. Lee, I. L. Kim, and S. Kyeong, "Fiber-optic waveguide coupled surface plasmon resonance sensor," Optics Express, 2012, 20(19): 21729-21738.

[15] B. Fan, L. Fang, Y. Li, X. Wang, K. Cui, F. Xue, et al., "Integrated refractive index sensor based on hybrid coupler with short range surface plasmon polariton and dielectric waveguide," Sensors and Actuators B: Chemical, 2003, 186: 495-505.

[16] W. R. Wong, O. Krupin, F. R. Mahamd Adikan, and P. Berini, "Optimization of long-range surface plasmon waveguides for attenuation-based biosensing," Journal of Lightwave Technology, 2015, 33(15): 3234-3242.

[17] J. Homola, J. Čtyroký, M. Skalský, J. Hradilová, and P. Kolářová, "A surface plasmon resonance based integrated optical sensor," Sensors 
and Actuators B: Chemical, 1997, 39(1-3): 286-290.

[18] R. D. Harris and J. S. Wilkinson, "Waveguide surface plasmon resonance sensors," Sensors and Actuators B: Chemical, 1995, 29(1-3): 261-267.

[19] L. Ji, S. Yang, R. Shi, Y. Fu, J. Su, and C. Wu, "Polymer waveguide coupled surface plasmon refractive index sensor: a theoretical study," Photonic Sensors, 2020, 10(4): 353-363.

[20] P. B. Johnson and R. W. Christy, "Optical constants of the noble metals," Physical Review B, 1972, 6(12): 4370-4379.

[21] O. Krupin, H. Asiri, C. Wang, R. N. Tait, and
P. Berini, "Biosensing using long-range surface plasmon-polariton waveguides," Optics Express, 2013, 21(1): 698-709.

[22] S. Kedenburg, M. Vieweg, T. Gissibl, and H. Giessen, "Linear refractive index and absorption measurements of nonlinear optical liquids in the visible and near-infrared spectral region," Optical Materials Express, 2012, 2(11): 15881611.

[23] Y. Xu, F. Wang, Y. Gao, D. Zhang, X. Sun, and P. Berini, "Straight long-range surface plasmon polariton waveguide sensor operating at $\lambda_{0}=$ 850 nm," Sensors, 2020, 20(9): 2507. 